\title{
ON THE $K$-THEORY OF CRYSTALLOGRAPHIC GROUPS
}

\author{
GEORGIOS TSAPOGAS
}

\begin{abstract}
For any crystallographic group $\Gamma$ we show that the groups $K_{i}(\Gamma)$ are isomorphic, via the forget control map, to the controlled $K$-groups $K_{i}(\Gamma)_{c}$, for all $i \leq 1$ and for an appropriate choice of the control map. By using this result and under a mild hypothesis on the crystallographic group $\Gamma$, it is proved that $K_{i}(\Gamma)=0$ for all $i \leq-2$ and $N^{j} K_{i}(\Gamma)=0$ for all $i \leq-1$ and $j>0$.
\end{abstract}

\section{INTRODUCTION}

In the solution to the Euclidean space form problem (see [11]), Farrell and Hsiang provided a vanishing theorem for $\mathrm{Wh}(\Gamma)$, where $\Gamma$ is a Bieberbach group, i.e. a torsion free crystallographic group. Later they showed the same result for torsion free poly-(cyclic or finite) groups (see [12]). For crystallographic groups though, a vanishing theorem is not plausible because $\Gamma$ can have finite subgroups which are retracts of it; and the Whitehead group of a finite group is very often nontrivial. Even the weaker statement $K_{-1}(\Gamma)=0$ is not plausible because Carter (see [3]) showed that, for a finite group $G, K_{-1}(G)$ is a finitely generated abelian group, whose rank is often nonzero.

For the same reasons the "nil" groups $N K_{1}(\Gamma)$ and $N K_{0}(\Gamma)$ will typically be nonzero for crystallographic groups, because $N K_{0}(\Gamma)$ is not zero for finite groups (see [1]). A central result of this paper, however (see Theorem 5.3 below), gives strong support for the following strengthening of a conjecture of W. C. Hsiang: If $\Gamma$ is a crystallographic group $N^{j} K_{i}(\Gamma)=0$, if $i=-1$ and $j>0$, or if $i \leq-2$ and $j \geq 0 .{ }^{1}$ In fact we prove:

Theorem 5.3. Let $\Gamma$ be a crystallographic group. Then $N^{j} K_{i}(\Gamma)=0$, if $i=-1$ and $j>0$, or if $i \leq-2$ and $j \geq 0$, provided that $\Gamma$ satisfies the following two properties:

(i) Each finite subgroup $H$ of $\Gamma / A_{C}$ is the image of a finite subgroup of $\Gamma$ under the natural epimorphism $\Gamma \rightarrow \Gamma / A_{C}$. Here $A_{C}$ is the sum of all the 1dimensional $G$-submodules of the translation subgroup $A$ of $\Gamma$, where $G$ is the holonomy subgroup.

(ii) If $\gamma \in \Gamma, a \in A$ and $\gamma a=a^{-1} \gamma$, then either $a=1$ or $\gamma$ has infinite order.

The first results concerning the $K$-theory of crystallographic groups are those of Quinn (see [19]), where he describes the $K$-theory of $\Gamma$ with rational co-

Received by the editors November 30, 1992.

1991 Mathematics Subject Classification. Primary 19D35; Secondary 19L64.

${ }^{1} \mathrm{~F}$. T. Farrell has posed the same conjecture for the class of finitely generated groups 
efficients as a homology theory with local coefficients in a $K$-theory spectrum. Yamasaki (see [27]) has proved an analogous theorem for the surgery groups of crystallographic groups. Moreover, Farrell and Jones obtained related results in [29].

The vanishing results for the Whitehead groups mentioned above have the following geometric significance: every $h$-cobordism on the corresponding manifold $M_{\Gamma}$ is a product, so that every manifold $h$-cobordant to $M_{\Gamma}$ is actually homeomorphic to it. Recently, F. Connolly and T. Kózniewski in their analysis of the equivariant structure set of $M_{\Gamma}$ (see [8]) provided the corresponding rigidity result for crystallographic manifolds. They showed that the Tate cohomology of $\mathbb{Z} / 2 \mathbb{Z}$ with coefficients in $\mathrm{Wh}_{G}^{\text {Top }, \rho}\left(M_{\Gamma}\right)$ vanishes. The latter group parametrizes the $G$-equivariant $h$-cobordisms on the flat crystallographic manifold $M_{\Gamma}$, where $G$ is the holonomy subgroup of $\Gamma$.

A crucial step in the course of the proof of Theorem 5.3 is the following:

Theorem 4.1. Let $\Gamma$ be any crystallographic group. Then the forget control map $F: K_{i}(\Gamma)_{c} \approx K_{i}(\Gamma)$ is an isomorphism for all $i \leq 1$, where the control space is $M_{\Delta} / G{ }^{2}$ Here $\Delta$ is a crystallographic group given as a quotient group of $\Gamma / A_{C}$ (see (2.1)), where $A_{C}$ is in Theorem 5.3, and $m_{\Delta}$ the flat crystallographic manifold associated to $\Delta$.

Theorem 4.1 combined with F. Quinn's results of [18] asserts that the $K$ theory of any crystallographic group $\Gamma$ is, in fact, an extraordinary homology theory with local coefficients. This fact is the key ingredient in the proof of Theorem 5.3.

\section{GROUP THEORETIC PRELIMINARIES}

In this section we will study crystallographic groups. $\Gamma$ will always denote a crystallographic group. We will define a crystallographic quotient group of $\Gamma$, denoted by $\Delta$. Then, using $\Delta$, we will establish the existence of expansive maps of $\Gamma$, analogous to the ones provided in [10], but suitable for our purposes. Moreover, the group $\Delta$ will be necessary in order to describe the control space for the groups $K_{i}(\Gamma)_{c}$ and carry out a hyperelementary induction argument needed in the proof of Theorem 4.1. We first recall some basic facts about crystallographic groups. (See [28] and [14].)

A crystallographic group $\Gamma$, of rank $n$, is a discrete cocompact subgroup of $E(n)$, the group of rigid motions of $\mathbb{R}^{n}$. The set of translations in $\Gamma$ forms a normal free abelian subgroup of $\Gamma$, which is called the translation subgroup of $\Gamma$ and is denoted by $A_{\Gamma}$. It is equal to its own centralizer. $G_{\Gamma}=\Gamma / A_{\Gamma}$ is a finite group called the holonomy group of $\Gamma$. A crystallographic group $\Gamma$ is uniquely determined by the resulting exact sequence

$$
0 \rightarrow A_{\Gamma} \rightarrow \Gamma \rightarrow G_{\Gamma} \rightarrow 1 .
$$

The precise algebraic characterization of crystallographic groups is the following (see [8]): Every group $\Gamma$ which contains a normal free abelian subgroup, which is equal to its own centralizer and has finite index, is isomorphic to a discrete subgroup of $E(n)$ which has compact quotient. The action of $\Gamma$ on $\mathbb{R}^{n}$ is factored into two steps: $A_{\Gamma}$ acts on $\mathbb{R}^{n}$ freely and the orbit space is a flat

\footnotetext{
${ }^{2}$ It was pointed out to the author by Professor Lawrence Taylor that 4.1 holds true for all $i \in \mathbb{Z}$.
} 
torus $\mathbb{R}^{n} / A_{\Gamma}$; the holonomy group $G_{\Gamma}$ acts on $\mathbb{R}^{n} / A_{\Gamma}$ as a group of isometries such that $\mathbb{R}^{n} / \Gamma \simeq G_{\Gamma} \backslash \mathbb{R}^{n} / A_{\Gamma}$. The torus $\mathbb{R}^{n} / A_{\Gamma}$ together with the $G_{\Gamma}$ action is called the crystallographic manifold associated to $\Gamma$ and it is denoted by $M_{\Gamma}$. It should be noted here that the notion of the crystallographic manifold associated to $\Gamma$ is understood, by several authors, in the following similar way: it is the pair $\left(\widetilde{M}_{\Gamma}, \Gamma\right)$ where $\widetilde{M}_{\Gamma}$ is the universal cover of $M_{\Gamma}$ and $\Gamma$ acts on $\widetilde{M}_{\Gamma}$ as a cocompact discrete group of isometries.

For all $s \in \mathbb{Z}$, let $\Gamma_{s}$ and $A_{s}$ denote $\Gamma / s A_{\Gamma}$ and $A_{\Gamma} / s A_{\Gamma}$ respectively. $\Gamma_{s}$ is again an extension of $G_{\Gamma}$ by $A_{s}$; i.e. the sequence

$$
0 \rightarrow A_{s} \rightarrow \Gamma_{s} \rightarrow G_{\Gamma} \rightarrow 0
$$

is exact. If $s$ is prime to $\left|G_{\Gamma}\right|$, then $A_{s} \rtimes G_{\Gamma} \approx \Gamma_{s}$. This is because the cohomology group $H^{2}\left(G_{\Gamma} ; A_{s}\right)$ vanishes. We will write $A_{\Gamma}$ and $G_{\Gamma}$ without subscripts when it is clear to which crystallographic group we refer. The following theorem was proved by Farrell and Hsiang (see [13]) and gives the structure of crystallographic groups.

Theorem A. Let $\Gamma$ be a crystallographic group with holonomy group $G$. Then either

(i) $\Gamma=\Delta \rtimes \mathbb{Z}$ for some nontrivial crystallographic group $\Delta$, with rank $\operatorname{rk}(\Delta)=$ $\mathrm{rk}(\Gamma)-1$, or

(ii) $\Gamma=B *_{D} C$ where $B, C$ and $D$ are crystallographic groups and $D$ has index 2 in both $B$ and $C$, or

(iii) there is an infinite sequence of positive integers $s$ with $s \equiv 1 \bmod \left(\left|G_{\Gamma}\right|\right)$, such that, any hyperelementary subgroup of $\Gamma_{s}$ which maps onto $G_{\Gamma}$ (via the natural map) is in fact isomorphic to $G_{\Gamma}$.

Recall that a hyperelementary group is an extension of a $p$-group by a cyclic group such that $(n, p)=1$. Here $n$ denotes the order of the cyclic group. The property described in case (iii) of the above theorem will be called for short "hypothesis $\mathscr{H}$ ".

The next proposition follows from Theorem A. It is proved by Farrell-Hsiang in [13].

Proposition B. Let $j: \Gamma \rightarrow \Delta$ be an epimorphism between crystallographic groups $\Gamma \subset E(n), \Delta \subset E(m)$. Then there exists a j-equivariant affine surjection $J: \mathbb{R}^{n} \rightarrow \mathbb{R}^{m}$. Moreover $j\left(A_{\Gamma}\right) \subseteq A_{\Delta}$, so that $j$ induces an epimorphism $j_{+}: G_{\Gamma} \rightarrow G_{\Delta}$.

It follows that there exists an equivariant map $p: M_{\Gamma} \rightarrow M_{\Delta}$, with fiber homeomorphic to the torus $T^{n-m}$.

(2.1) Let now $\Gamma$ be an arbitrary crystallographic group. If $\Gamma$ satisfies case (i) of Theorem $A$, then $\Gamma$ admits an epimorphism to $\mathbb{Z}$, which is a trivial 1dimensional $G$-submodule of $A_{\Gamma}$ contained in the center of $\Gamma$. In case (ii) of Theorem $\mathrm{A}, \Gamma$ admits an epimorphism $f: \Gamma \rightarrow D$. Here $D$ denotes the infinite dihedral group, which is a crystallographic group with corresponding exact sequence $0 \rightarrow \mathbb{Z} \rightarrow D \rightarrow \operatorname{Aut}(\mathbb{Z}) \rightarrow 1$. The map $f$ induces an epimorphism $\omega: G_{\Gamma} \rightarrow \operatorname{Aut}(\mathbb{Z}) \approx\{ \pm 1\}$. Let $C$ denote the image of $\operatorname{Hom}(\Gamma, D)$ in $\operatorname{Hom}(G,\{ \pm 1\})$. For each $\omega \in C$ let $A^{\omega}=\left\{a \in A_{\Gamma} \mid g \cdot a=\omega(g) \cdot a \forall g \in G\right\}$. Note that, for $\omega$ trivial, $A^{\omega}$ is just the center of $\Gamma$. Set $A_{C}=\sum_{\omega \in C} A^{\omega}$. It is obvious that $A_{C}$ is normal in $\Gamma$. Let $\tilde{\Delta}=\Gamma / A_{C}, \tilde{j}: \widetilde{\Delta} \rightarrow G$ be the 
map induced by the epimorphism $j: \Gamma \rightarrow G$ and $p: G \rightarrow \operatorname{Aut}\left(A / A_{C}\right)$ be the natural map. Define $\Delta$ to be the quotient group $\widetilde{\Delta} / T$, where $T$ denotes the subgroup of $\tilde{j}^{-1}(\operatorname{ker} p)$ which consists of all the elements of finite order in $\tilde{j}^{-1}(\operatorname{ker} p)$. Note that $\operatorname{ker} p$ is an elementary abelian 2-group. It can be shown (see [26]) that $\Delta$ is a crystallographic group with translation subgroup $\tilde{j}^{-1}(\operatorname{ker} p) / T \approx A / A_{C}$ and holonomy group $G_{\Delta}$ which fits into the exact sequence $1 \rightarrow \operatorname{ker} p \rightarrow G \rightarrow G_{\Delta} \rightarrow 1$. Moreover, the crystallographic group $\Delta$ satisfies hypothesis $\mathscr{H}$ (for hypothesis $\mathscr{H}$ see Theorem A).

We now proceed to describe a specific type of expansive map which will be needed in the proof of Theorem 4.1. If $\Gamma$ is a crystallographic group, a monomorphism $f: \Gamma \rightarrow \Gamma$ is said to be $s$-expansive if it is multiplication by $s$ on the translation subgroup $A$ and induces the identity on $G$. D. Epstein and $M$. Shub showed in [10] that compact manifolds with a flat Riemannian metric admit expanding endomorphisms, i.e. torsion free crystallographic groups admit expansive maps.

(2.2) Let $\sigma$ be the element $\sigma=\sum_{\omega \in C} \sigma_{\omega} \in \operatorname{Hom}_{G}\left(A_{\Gamma}, A_{\Gamma}\right)$, where $\sigma_{\omega}=$ $\sum_{g \in G} \omega(g)^{-1} g$. For each integer $m \geq 0$ set $\psi=m\left(|G| \mathrm{id}_{A_{\Gamma}}-\sigma\right)$. The order of $G$ annihilates $H^{2}\left(G ; A_{\Gamma}\right)$ and therefore, there exists a crossed homomorphism $h: \Gamma \rightarrow A_{\Gamma}$ representing an element of $H^{1}\left(\Gamma ; A_{\Gamma}\right)$ such that $\left.h\right|_{A_{\Gamma}}=|G| \psi$. For each $s=1+m|G|^{2}$, define

$$
f_{s}: \Gamma \rightarrow \Gamma: f_{s}(\gamma)=h(\gamma) \cdot \gamma
$$

$f_{s}$ is a monomorphism such that $f_{s}\left(A_{\Gamma}\right) \subseteq A_{\Gamma}, f_{s \mid A_{C}}$ is the identity and induces multiplication by $s$ on $A_{\Gamma} / A_{C}$. Moreover $f_{s}$ induces a map on $\widetilde{\Delta}$ which in turn gives rise to an expansive endomorphism $g_{s}: \Delta \rightarrow \Delta$.

(2.4) It can be shown (see [26]) that $f_{s}$ and $g_{s}$ induce an $f_{s}$-equivariant diffeomorphism $F_{s}$ of Euclidean $n$-space and a $g_{s}$-equivariant diffeomorphism $G_{s}$ of Euclidean $(n-k)$-space such that $\left|d G_{s}(X)\right|=s|X|$ for all tangent vectors $X$ of Euclidean space and the following diagram commutes:

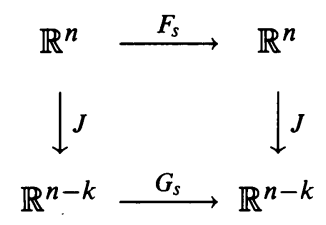

Here $k$ is the rank of $A_{C}$ and $J$ is the map provided by Proposition B using the epimorphism $j: \Gamma \rightarrow \Delta$. Note that if $A_{C}$ is trivial, then the maps described above reduce to the expansive maps given in [10]. But if $A_{C} \neq 0$, then $f_{s}$ expands distances in the directions perpendicular to the fibers of the map $p: M_{\Gamma} \rightarrow M_{\Delta}$.

\section{GeOMETRIC PRELIMINARIES}

In this section we will state the results of F. Quinn (see [18]) adapted to our needs. We will also give an alternative definition for the $K$-theory, following R. Stöcker (see [23]), which will be suitable for applying the results of [18].

The Whitehead group $\mathrm{Wh}(\pi)$ of a group $\pi$, as well as $K_{0}(R)$ for a ring $R$, is classical and they are defined in various places (see for example [15] and [17]). The lower $K$-groups $K_{-i}(\mathbb{Z} \pi)$ were first defined by Bass in [1] and later by Ranicki as transfer invariant elements of $\mathrm{Wh}\left(\pi \times \mathbb{Z}^{i+1}\right)$ (see [21]). 
The Whitehead group $\mathrm{Wh}(X)$ of a CW complex $X$ is defined geometrically in various places (see [6]). A typical element in $\mathrm{Wh}(X)$ is an equivalence class of pairs $(Y, f)$, where $Y$ is a CW complex and $f: Y \rightarrow X$ is a strong deformation retraction. It is well known that the expansion and collapsing maps, which generate the equivalence relation in the original definition of $\mathrm{Wh}(X)$, can be replaced by maps with contractible point inverses (see [7]). Finally, Chapman in [5] defines the controlled Whitehead group $\mathrm{Wh}(X)_{c}$ of a CW complex $X$ equipped with a map $p: X \rightarrow B, B$ a metric space, as the inverse limit $\mathrm{Wh}(X)_{c} \equiv{\underset{\lim }{\longleftarrow}}_{\varepsilon} \mathrm{Wh}(X)_{\varepsilon}$, where $\mathrm{Wh}(X)_{\varepsilon}$ consists of all the pairs $(Y, f)$ for which the homotopy corresponding to the retraction is $\varepsilon$-controlled. Then the lower controlled $K$-groups $K_{-i}(X)_{c}$ are defined as subgroups of $\mathrm{Wh}\left(X \times T^{i+1}\right)_{c}$, consisting of all elements invariant under transfers induced by expanding covers $f: T^{i+1} \rightarrow T^{i+1}$.

In all of the above definitions for the Whitehead group $\mathrm{Wh}(X)$, assume that $X$ is a finite $\mathrm{CW}$ complex or at least locally compact $\mathrm{CW}$ complex. In particular, the same holds true for the controlled groups. However, one can define the Whitehead group of an arbitrary CW complex following Stöcker (see [23]):

Let $X$ be any CW complex. Consider all pairs $(Y, f)$ where $(Y, X)$ is a finite relative $\mathrm{CW}$ complex and $f: Y \rightarrow X$ a strong deformation retraction. Two such elements $\left(Y_{1}, f_{1}\right)$ and $\left(Y_{2}, f_{2}\right)$ are said to be equivalent provided that there exist a CW complex $Z$ containing $X$ and maps $g_{1}: Z \rightarrow Y_{1}, g_{2}: Z \rightarrow Y_{2}$, each one of which is a finite sequence of elementary expansions and collapses, such that the following diagram commutes up to homotopy $\operatorname{rel} X$ :

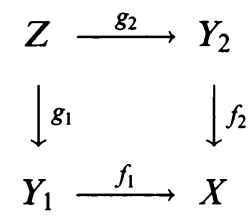

Then the Whitehead group consists of equivalence classes of such pairs. Moreover, as in the finite case, $\mathrm{Wh}(X) \approx \mathrm{Wh}\left(\pi_{1}(X)\right)$. This description of $\mathrm{Wh}(X)$ is suitable for carrying over the construction given in [6] for $\mathrm{Wh}(X)_{c}$; i.e. the definition of $\mathrm{Wh}(X)_{c}$, for an arbitrary $\mathrm{CW}$ complex $X$, is completely analogous with the finite case. We proceed now to outline the definition of the controlled $K$-theory of a crystallographic group, which we will use in this paper.

(3.1) Let $\Gamma$ be a crystallographic group with holonomy group $G$ and $M_{\Gamma}$ the crystallographic manifold associated to $\Gamma$. Consider $M_{\Gamma} \times_{G} E G \rightarrow B G$, the associated bundle over $B G$ with fiber $M_{\Gamma}$. Here $B G$ is an EilenbergMac Lane space of type $K(G, 1)$ and $E G$ is contractible with $G$ acting freely on $E G$ (see [16]). The universal cover of $M_{\Gamma} \times_{G} E G$ is $\widetilde{M}_{\Gamma} \times E G$, where $\widetilde{M}_{\Gamma}$ is the universal cover of $M_{\Gamma} . \Gamma$ acts diagonally on $M_{\Gamma} \times E G$, the action is free and $\left(\widetilde{M}_{\Gamma} \times E G\right) / \Gamma \approx M_{\Gamma} \times_{G} E G$. So we have $\pi_{1}\left(M_{\Gamma} \times_{G} E G\right) \approx \Gamma$. Therefore we can identify $K_{i}(\Gamma)$ with $K_{i}\left(M_{\Gamma} \times_{G} E G\right)$ and we will write $K_{i}(\Gamma)_{c}$ for the controlled group $K_{i}\left(M_{\Gamma} \times_{G} E G\right)_{c}$, for all $i \leq 1$, where the control map is given by the composition

$$
M_{\Gamma} \times_{G} E G \stackrel{\operatorname{id}_{M} \times c}{\longrightarrow} M_{\Gamma} \times_{G} * \approx M_{\Gamma} / G \rightarrow M_{\Delta} / G
$$


Here the map $c$ is just the constant map and the second map is provided by Proposition B.

A crucial result for this paper is due to F. Quinn. Roughly speaking, in Chapter 8 of [18], F. Quinn discusses (generalized) homology with "twisted" spectrum coefficients and he shows the existence of an Atiyah-Hirzebruch type spectral sequence which relates it to ordinary homology. In the next paragraph we specialize this result for our case.

(3.3) We will restrict our attention to the $K$-theory functor, denoted by $\mathbb{K}$. This is a functor from spaces to spectra (constructed in [18]), such that for any space $X, \mathbb{K}(X)$ is a (nonconnective) spectrum whose homotopy groups are the ordinary $K$-groups $K_{i}(X)$.

Let $p$ be the projection $M_{\Gamma} \times_{G} E G \rightarrow M_{\Delta} / G_{\Delta}$, which is the control space for $K_{i}(\Gamma)_{c}$, and let $p_{\Gamma}$ denote $M_{\Gamma} \times_{G} E G \rightarrow M_{\Gamma} / G_{\Gamma}$. We will denote elements of $M_{\Gamma} / G_{\Gamma}$ or $M_{\Delta} / G_{\Delta}$ by $[x]$ and elements of $\mathbb{R}^{n}=O(n) \backslash E(n)$ representing $[x]$ by $x$. Let $\mathbb{K}(p)$ denote the sheaf obtained by applying $\mathbb{K}$ to the stalks $p^{-1}([x])$.

Homology groups with coefficients in $\mathbb{K}(p), \mathbf{H}_{i}\left(M_{\Delta} / G_{\Delta} ; \mathbb{K}(p)\right)$, are defined in $[18, \S 8.1]$. In our case, i.e. when the $K$-theory spectrum $\mathbb{K}$ together with the control map $p: M_{\Gamma} \times_{G} E G \rightarrow M_{\Delta} / G_{\Delta}$ is used, these homology groups are just Quinn's alternative definition for $K_{i}(\Gamma)_{c}$ (see [20]). Then from [18, §8.7], we have that there exists an Atiyah-Hirzebruch spectral sequence $E_{\Gamma}$ with $E^{2}$ term given by $E_{j, i-j}^{2}=H_{j}^{G}\left(M_{\Delta}: \pi_{i-j} \mathbb{K}\left(p^{-1}(x)\right)\right)$ which abuts to $K_{i}(\Gamma)_{c}$. The coefficient groups $\pi_{i} \mathbb{K}\left(p^{-1}([x])\right)$ are just $K_{i}\left(\pi_{1}\left(p^{-1}([x])\right)\right)$.

The homology theory used here is $G$-equivariant homology with respect to the homology coefficient system given by the functors $K_{i}: O_{G} \rightarrow \mathrm{Abel}$, where $O_{G}$ is the category whose objects are subgroups of $G$ and morphisms are inclusions or conjugations, and $A b e l$ is the category of abelian groups as usual. This is the homology analogue of Bredon's equivariant cohomology theory. It is explained in (3.5) below.

The next step is to examine $p^{-1}([x])$-especially $\pi_{1}\left(p^{-1}([x])\right)$-where $[x]$ is an element of $M_{\Delta} / G_{\Delta}$. First note that if $x \in O(n) \backslash E(n)=\mathbb{R}^{n}$ is a representative of the orbit $\Gamma \cdot x=[x] \in M_{\Gamma} / G_{\Gamma}$, then the isotropy subgroup $G_{x}=\Gamma \cap\left(x O(n) x^{-1}\right)$, being a discrete set in a compact space, is finite. For $[x] \in M_{\Gamma} / G_{\Gamma}$ we have the following calculation:

$$
p_{\Gamma}^{-1}([x])=G \cdot x \times_{G} E G \simeq G / G_{x} \times_{G} E G \simeq E G / G_{x} \simeq B G_{x},
$$

so that $\pi_{1}\left(p_{\Gamma}^{-1}([x])\right)=G_{x}$. Since the control is $p: M_{\Gamma} \times_{G} E G \rightarrow M_{\Delta} / G_{\Delta}$, we are interested in the fundamental group of the stalks $p^{-1}([x])$ for $[x] \in M_{\Delta} / G_{\Delta}$.

Lemma 3.4. $\pi_{1}\left(p^{-1}([x])\right)$ is an extension of $\left(G_{\Delta}\right)_{x}$ by a free abelian group of rank less than $\operatorname{rk}(\Gamma)$. Moreover, $\pi_{1}\left(p^{-1}([x])\right) \approx A_{C} \times\left(G_{\Delta}\right)_{x}$, if the following conditions hold:

(i) Each finite subgroup $H$ of $\Gamma / A_{C}$ is the image of a finite subgroup of $\Gamma$ under the natural epimorphism $\Gamma \rightarrow \Gamma / A_{C}$. Here $A_{C}$ is the sum of all the 1-dimensional $G$-submodules of the translation subgroup of $\Gamma$.

(ii) If $\gamma \in \Gamma, a \in A$ and $\gamma a=a^{-1} \gamma$, then either $a=1$ or $\gamma$ has infinite order.

Proof. Consider $T^{k} \times_{G} E G \rightarrow B\left(G_{\Delta}\right)_{x}$, the associated bundle over $B\left(G_{\Delta}\right)_{x}$, with fiber the $k$-torus. Here $k$ is the rank of the free abelian group $A_{C}$ which 
was used to define $\Delta$ from $\Gamma$ (cf. (2.1)). The homotopy exact sequence of this bundle shows that $\pi_{1}\left(p^{-1}([x])\right)$ fits into a short exact sequence

$$
0 \rightarrow \pi_{1}\left(T^{k}\right) \rightarrow \pi_{1}\left(p^{-1}([x])\right) \rightarrow \pi_{1}\left(B\left(G_{\Delta}\right)_{x}\right) \rightarrow 0,
$$

where the action of $G$ on $A_{\Gamma}$ determines the $\left(G_{\Delta}\right)_{x}$-module structure of $A_{C} \approx$ $\pi_{1}\left(T^{k}\right)$. By condition (ii), $\left(G_{\Delta}\right)_{x}$ acts trivially on $A_{C}$; i.e. the image of $\pi_{1}\left(T^{k}\right)$ in $\pi_{1}\left(p^{-1}([x])\right)$ is central. By condition (i) the above exact sequence splits so that $\pi_{1}\left(p^{-1}([x])\right) \approx A_{C} \times\left(G_{\Delta}\right)_{x}$.

(3.5) We conclude this section by giving an outline of the homology analogue of Bredon's equivariant cohomology theory (see [2]). Let $G$ be a finite group and let $O_{G}$ be the category whose objects are subgroups of $G$ and morphisms are objects as follows: if $H, K$ are subgroups of $G$, then a morphism from $H$ to $K$ is a coset $K g$ such that $H \subseteq{ }^{g} K$. Composition between two morphisms $K g$ and $L g^{\prime}$ is given by $\left(L g^{\prime}\right)(K g)=\left(g^{\prime} g\right) L$. A homology coefficient system is a covariant functor $F: O_{G} \rightarrow \mathrm{Abel}$, where $\mathrm{Abel}$ is the category of abelian groups. If $g \in G, H$ is a subgroup of $G$ and $a \in F\left({ }^{g} K\right)$ we will write $a \cdot g$ for $F(H \stackrel{H g}{\rightarrow} g K)(a)$. Observe that $a \cdot\left(g_{1} g_{2}\right)=\left(a \cdot g_{1}\right) \cdot g_{2}$.

Let $X$ be a $G$-equivariant $C W$ complex, where the cells are oriented in such a way that $G$ preserves orientation. Let $\sigma$ be an $n$-cell. For each $n-1$ cell $\tau$, let $[\sigma: \tau]$ be the degree of the map $f_{\sigma}^{\tau}: S^{n-1} \rightarrow \tau / \dot{\tau}$, obtained from the attaching map $f_{\sigma}: S^{n-1} \rightarrow X^{n-1}$, after collapsing the $n-2$ skeleton to a point. If $[\sigma: \tau] \neq 0$, we get an inclusion $G_{\sigma} \subset G_{\tau}$ which induces a map $(\sigma \rightarrow \tau)_{*}: F\left(G_{\sigma}\right) \rightarrow F\left(G_{\tau}\right)$. Here $G_{\sigma}$ denotes the stabilizer of $\sigma$. An equivariant $n$-chain is a formal sum $\sum a_{\sigma} \cdot \sigma$, where $a_{\sigma} \in F\left(G_{\sigma}\right)$, provided that the relation $a \cdot g \sigma=(a \cdot g) \cdot \sigma$ is satisfied. Denote by $C_{n}^{G}(X ; F)$ the group of all $n$-chains on $X$. The map $E^{G}: C_{n}^{G}(X ; F) \rightarrow C_{n-1}^{G}(X ; F)$ defined by the equation $E^{G}(a \cdot \sigma)=\sum_{\tau}[\sigma: \tau]\left((\sigma \rightarrow \tau)_{*} a\right) \tau$ makes $\left(C_{*}^{G}(X ; F), E^{G}\right)$ into a chain complex. Define the equivariant homology groups with coefficients in $F$ to be the homology groups of this chain complex, i.e. $H_{n}^{G}(X: F) \equiv H_{n}\left(C_{*}^{G}(X ; F)\right)$. If we specialize $F$ to be the coefficient system which assigns to each subgroup $H$ of $G$ the abelian group $K_{i}(\mathbb{Z} H)$ and to each inclusion $H \subset K$ the induction map $\operatorname{res}_{H}^{K}: K_{i}(\mathbb{Z} H) \rightarrow K_{i}(\mathbb{Z} K)$, then we obtain the homology theory used in the spectral sequence mentioned in (3.3) above.

\section{THE FORGET CONTROL ISOMORPHISM}

Let $\Gamma$ be any crystallographic group and $\Delta$ the crystallographic group constructed in (2.1). In this section we show that the $K$-theory groups $K_{i}(\Gamma)$ are isomorphic to the controlled groups $K_{i}(\Gamma)_{c}$. We will be viewing $K_{i}(\Gamma)$ geometrically as $K_{i}\left(M \times{ }_{G} E G\right)$, as explained in (3.1), and we write $K_{i}(\Gamma)_{c}$ for the controlled group $K_{i}\left(M_{\Gamma} \times_{G} E G\right)_{c}$, where the control map is $M_{\Gamma} \times_{G} E G \rightarrow M_{\Delta} / G$, as described in (3.2). We now state the main theorem of this section:

Theorem 4.1. Let $\Gamma$ be any crystallographic group. Then the forget control map $F: K_{i}(\Gamma)_{c} \stackrel{\approx}{\rightarrow} K_{i}(\Gamma)$ is an isomorphism for all $i \leq 1$, where the control space is $M_{\Delta} / G$.

An important tool which will be used for the proof of Theorem 4.1 is Dress induction, which implies that the $K$-functors are computable from the category of hyperelementary subgroups of the given group. Recall that a hyperelementary 
group is an extension of a $p$-group by a cyclic group of order $n$ such that $(n, p)=1$. In the next paragraph we will briefly state this induction theorem.

(4.2) Let $j: \Gamma \rightarrow G$ be a homomorphism from a group $\Gamma$ to a finite group $G$. For each subgroup $H$ of $G$, denote by $\Gamma_{H}$ the inverse image $j^{-1}(H)$. Let $G_{0}(\mathbb{Z} H)$ denote Swan's ring, i.e. the Grothendieck ring of left $\mathbb{Z} H$-modules which are finitely generated and free as $\mathbb{Z}$-modules (see [24]). It is well known that the functors $K_{i}\left(\Gamma_{(-)}\right)$are Green modules over $G_{0}(\mathbb{Z}(-))$ : if $[M] \in G_{0}(\mathbb{Z} H)$ and $[P, \alpha] \in K_{1}\left(\Gamma_{H}\right)$, the rule $[M] \cdot[P, \alpha] \rightarrow\left[P \otimes_{\mathbb{Z}} M, \alpha \otimes_{\mathbb{Z}} \mathrm{id}_{M}\right]$ respects relations and makes $K_{1}\left(\Gamma_{H}\right)$ a module over $G_{0}(H)$-for details see [13].

Let $\mathscr{K}$ denote the category whose objects are the hyperelementary subgroups of $G$ and a map between two subgroups $H$ and $K$ is an element $g \in G$, such that $\mathrm{gHg}^{-1} \subseteq K$. Dress's theorem asserts that $K_{i}(\Gamma)$ is $\mathscr{K}$ computable, i.e.

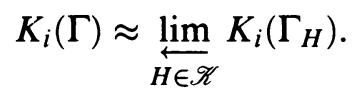

Recall that

$$
\begin{array}{r}
\lim _{H \in \mathscr{K}} K_{i}\left(\Gamma_{H}\right)=\left\{\left(g_{\Gamma_{H}}\right)_{H \in \mathscr{K}} \in \prod_{H \in \mathscr{K}} K_{i}\left(\Gamma_{H}\right) / c_{g}^{*}\left(g_{\Gamma_{H}}\right)=\left(g_{\Gamma_{K}}\right)\right. \\
\left.\quad \text { for all } H, K \text { and } c_{g} \in \operatorname{Map}_{\mathscr{K}}(H, K)\right\} .
\end{array}
$$

Connolly and Kózniewski in [8] prove that the controlled $K$-theory is again computable from $\mathscr{K}$. They show by Dress induction (see A. Dress [9]) that $K_{i}(\Gamma)_{c} \approx \lim _{H \in \mathscr{K}} K_{i}\left(\Gamma_{H}\right)_{c}$.

We will also need the following lemma which is a weak version of Proposition 2.9 in [8].

Lemma 4.3. Let $\Gamma$ be any crystallographic group. For each $i \leq 1$, there exists a number $\varepsilon>0$, such that the relax control map $K_{i}(\Gamma)_{c} \rightarrow K_{i}(\Gamma)_{\varepsilon}$ is injective.

Proof. As a first step we claim that $K_{i}(\Gamma)_{c}$ is countable. This follows from the existence of the spectral sequence, mentioned in (3.3) above, provided we can show that $E_{j, i-j}^{2}$ is countable for $j \geq 0$. So it is enough to show that the coefficient groups, $K_{i}\left(\pi_{1}\left(p_{\Delta}^{-1}(x)\right)\right)$, for the $E^{2}$ term are countable. But by Lemma 3.4 we have that the groups $\pi_{1}\left(p_{\Delta}^{-1}(x)\right)$ are finitely generated and therefore, for each integer $n, \mathrm{GL}_{n}\left(\mathbb{Z} \pi_{1}\left(p_{\Delta}^{-1}(x)\right)\right)$ is countable. Then, $\operatorname{GL}\left(\mathbb{Z} \pi_{1}\left(p_{\Delta}^{-1}(x)\right)\right)$ is countable because it is the ascending union of countable sets. Hence $K_{i}(\Gamma)_{c}$ is countable for each $i \leq 1$.

The second step is to give each group $K_{i}(\Gamma)_{\varepsilon}$ the discrete metric. Then $K_{i}(\Gamma)_{c}$ is closed as a subgroup of the direct product $\prod K_{i}(\Gamma)_{1 / n}$. So $K_{i}(\Gamma)_{c}$ is a complete metric space. According to the Baire category theorem, at least one point in $K_{i}(\Gamma)_{c}$ must be open. Since $K_{i}(\Gamma)_{c}$ is a topological group, we may assume that 0 is open. By the definition of the product topology there are finitely many open neighborhoods $U_{j}$ of 0 in $K_{i}(\Gamma)_{1 / n_{j}}$ such that

$$
K_{i}(\Gamma)_{c} \cap\left(U_{1} \times U_{2} \times \cdots \times U_{k} \times \prod_{j \neq 1, \ldots, k} K_{i}(\Gamma)_{1 / n}\right)=\{0\} .
$$


Replace these $U_{k}$ by smaller open sets, if necessary, so that $U_{k}$ is mapped into $U_{k+1}$ under the relax control map. So if $\varepsilon=1 / n_{1}$ and if $\rho: K_{i}(\Gamma)_{c} \rightarrow K_{i}(\Gamma)_{\varepsilon}$ is the relax control map, $\rho^{-1} U_{1}=\{0\}$. So $\rho: K_{i}(\Gamma)_{c} \rightarrow K_{i}(\Gamma)_{\varepsilon}$ is injective.

Proof of Theorem 4.1. Let $\Gamma$ be any crystallographic group of rank say $n$ and holonomy group $G$. Fix $i \leq 1$. Assume that the conclusion of the theorem is true for all crystallographic groups whose rank is less than $n$ or whose rank is $n$ and whose holonomy group has order less than $\left|G_{\Gamma}\right|$. Let $\Delta_{s}$ denote $\Delta / s A_{\Delta}$, where $s \equiv 1 \bmod \left(\left|G_{\Delta}\right|^{2}\right)$. Let $j$ be the epimorphism $\Gamma \rightarrow \Delta \rightarrow 0$, where $\Delta$ is the group defined in (2.1); let $j_{s}$ denote the natural map $\Delta \rightarrow \Delta_{s}$. Denote by $\mathscr{K}$ the class of hyperelementary subgroups of $\Delta_{s}$. By Dress induction we have

$$
K_{i}(\Gamma) \approx \lim _{H \in \mathscr{K}} K_{i}\left(\Gamma_{H}\right) \text { and } K_{i}(\Gamma)_{c}=\underset{H \in \mathscr{K}}{\lim } K_{i}\left(\Gamma_{H}\right)_{c}
$$

If $G_{\Delta}$ is not hyperelementary, then, since $\Delta$ satisfies hypothesis $\mathscr{H}$, any hyperelementary subgroup of $\Delta_{s}$ which surjects onto $G_{\Delta}$ is isomorphic to $G_{\Delta}$. Therefore, for all $s$ as above, all groups $\Gamma_{H}$ have holonomy groups of order less than $\left|G_{\Gamma}\right|$. Hence by the induction hypothesis the forget control maps $F_{H}$ : $K_{i}\left(\Gamma_{H}\right)_{c} \rightarrow K_{i}\left(\Gamma_{H}\right)$ are isomorphisms. The result now follows by taking the inverse limit of these isomorphisms $F_{H}$ to conclude that the forget control map $F: K_{i}(\Gamma)_{c} \rightarrow K_{i}(\Gamma)$ is an isomorphism.

Suppose now that $G_{\Delta}$ is hyperelementary. Recall that, for all $s$ as above, $\left|\left(A_{\Delta}\right)_{s}\right|$ and $\left|G_{\Delta}\right|$ are relatively prime, so that $H^{q}\left(G ;\left(A_{\Delta}\right)_{s}\right)=0, q=1,2$; i.e. the sequence $0 \rightarrow\left(A_{\Delta}\right)_{s} \rightarrow \Delta_{s} \rightarrow G_{\Delta} \rightarrow 1$ has a splitting $\sigma: G_{\Delta} \rightarrow \Delta_{s}$. $\sigma$ is unique up to conjugacy. For each $s$ as above let $f_{s}: \Gamma \rightarrow \Gamma$ be the monomorphism (2.3).

Claim. $f_{s}(\Gamma)=\Gamma_{H_{1}}$ where $H_{1}$ is conjugate to $\sigma\left(G_{\Delta}\right)$.

Proof of the claim. The monomorphism $f_{s}$ induces the following commutative diagram:

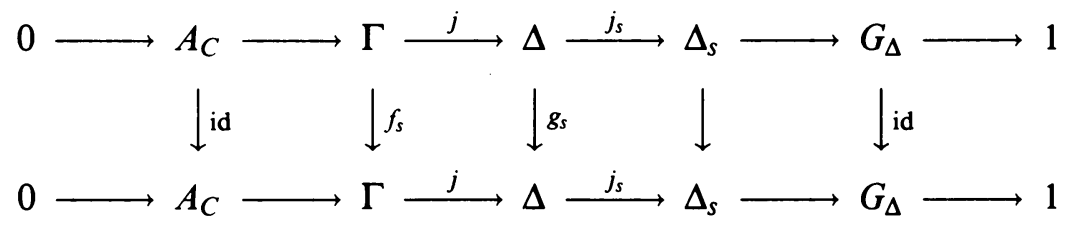

Moreover, $g_{s}$ is an expanding endomorphism for the crystallographic group $\Delta$ in the sense of Epstein-Shub in [10] (cf. (2.4)). So $g_{s}$ induces the following commutative diagram:

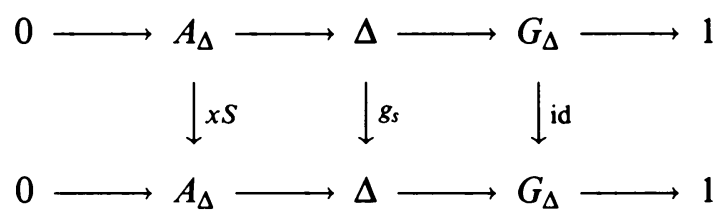

Since $g_{s}$ induces the zero map on $\left(A_{\Delta}\right)_{s}$, diagram (4.5) implies that $j_{s}^{-1}\left(H_{1}\right)=$ $g_{s}(\Delta)$, for some $H_{1}$ surjecting onto $G_{\Delta}$. But then, by diagram (4.4) above,

$$
f_{s}(\Gamma)=j^{-1}\left(g_{s}(\Delta)\right)=j^{-1} j_{s}^{-1}\left(H_{1}\right)=\Gamma_{H_{1}} .
$$

This completes the proof of the claim. 
Hence the $H_{1}$ coordinate of the restriction map $K_{i}(\Gamma) \rightarrow \stackrel{\lim }{\longleftarrow}_{H \in \mathscr{H}} K_{i}\left(\Gamma_{H}\right)$ is given by $f_{s}^{*}$; i.e. the map $K_{i}(\Gamma) \stackrel{f_{s}^{*}}{\rightarrow} K_{i}\left(\Gamma_{H_{1}}\right)$ is an isomorphism onto its image. Let $x$ be an element in $K_{i}(\Gamma)$. As we saw earlier in (3.1), $x$ can be considered as an element $K_{i}\left(M_{\Gamma} \times_{G} E G\right)$. Represent $x$ by a strong deformation retraction $h: Y \rightarrow M_{\Gamma} \times_{G} E G$. Let $\delta(x)$ denote the control of this element; i.e. $\delta(x)=\max _{y \in Y}\left\{\operatorname{diam}\left\{p\left(h_{t}(y)\right) \mid t \in[0,1]\right\}\right.$, where $h_{t}$ is the homotopy associated to $x$ and $p$ is the control map $M \times_{G} E G \rightarrow M_{\Delta} / G$.

We first show that the map $F: K_{i}(\Gamma)_{c} \rightarrow K_{i}(\Gamma)$ is injective. Let $x \in \operatorname{Ker} F$. Then $x$ can be represented by an element $(Y, h)$, where $h: Y \rightarrow M_{\Gamma} \times_{G} E G$ is a strong deformation retraction $\operatorname{rel} X$, such that there exist a CW complex $Z$ containing $M_{\Gamma} \times_{G} E G$ and $C E$ maps $Z \rightarrow M_{\Gamma} \times_{G} E G, Z \rightarrow Y$ making the diagram

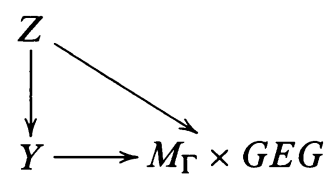

commute up to (uncontrolled) homotopy. We wish to show that $x$ is zero in $K_{i}(\Gamma)_{c}$. Let $\varepsilon$ be the positive number provided by Lemma 4.3 above. It is enough to show that, for all $H \in \mathscr{K}$, $\operatorname{res}_{H}^{G}(x)=0$ in $K_{i}\left(\Gamma_{H}\right)_{c}$. If $H$ is not conjugate to $H_{1}$, then $H$ does not surject onto $G$ and therefore $\operatorname{rk}\left(\Gamma_{H}\right) \leq$ $\operatorname{rk}(\Gamma)$. By induction hypothesis $F_{H}: K_{i}\left(\Gamma_{H}\right)_{c} \rightarrow K_{i}\left(\Gamma_{H}\right)$ is an isomorphism, which implies that $\operatorname{res}_{H}^{G}(x)=0$ in $K_{i}\left(\Gamma_{H}\right)_{c}$. If $H=H_{1}, \operatorname{res}_{H}^{G}(x)=f_{s}^{*}(x)$ because $f_{s}(\Gamma)=\Gamma_{H}$. But then the diagram (4.6) commutes up to $\delta(x) / s$ homotopy, because $f_{s}^{*}$ expands distances on $M_{\Delta}$, by a factor of $s$. So for all $s$ sufficiently large, namely $s>\delta(x) / \varepsilon, f_{s}^{*}(x)$ lies in the image of the composite map

$$
K_{i}(\Gamma)_{c} \stackrel{f_{s}^{*}}{\rightarrow} K_{i}(\Gamma)_{c} \stackrel{\rho}{\rightarrow} K_{i}(\Gamma)_{\varepsilon} .
$$

Since by Lemma $4.3 \rho$ is injective, this implies that $x \in \operatorname{ker} f_{s}^{\#}$; i.e. $x$ belongs to the kernel of $\operatorname{res}_{H}^{G}$. Since $x \in \operatorname{Ker}\left(\operatorname{res}_{H}^{G}\right)$ for all $H$ in $\mathscr{K}$, we conclude $x=0$. This completes the proof that $F$ is injective.

Next we show that the map $F: K_{i}(\Gamma)_{c} \rightarrow K_{i}(\Gamma)$ is surjective. Here we will use Quinn's stability result (see [18, Theorem 4.1$]$ ) which states that the inverse limit $\lim _{\varepsilon} K_{i}(\Gamma)_{\varepsilon}$ is stable. This means that for each element $x \in K_{i}(\Gamma)$ there exists $\varepsilon_{0}=\varepsilon_{0}(x)>0$ such that if $x$ belongs to the image of the map $F_{\varepsilon_{0}}: K_{i}(\Gamma)_{\varepsilon_{0}} \rightarrow$ $K_{i}(\Gamma)$, then $x \in \operatorname{Im} F$. Let $x$ be an arbitrary element in $K_{i}(\Gamma)$. The argument will be almost identical with the one given in the proof of the injectivity of $F$. By Dress induction it suffices to check that $\operatorname{res}_{H}^{G}(x) \in \operatorname{Im} F_{H}$ for all $H \in \mathscr{K}$. If $H$ is not conjugate to $H_{1}$, then, as before, by induction hypothesis, $F_{H}$ is an isomorphism and therefore $\operatorname{res}_{H}^{G}(x) \in \operatorname{Im} F_{H}$. If $H$ is conjugate to $H_{1}$, let $\varepsilon_{0}$ be the number which corresponds to the element $\operatorname{res}_{H}^{G}(x)=f_{s}^{\#}(x) \in K_{i}\left(\Gamma_{H}\right)$, given by the stability of the inverse limit $\lim _{\varepsilon} K_{i}\left(\Gamma_{H}\right)_{\varepsilon}$. It is enough to show that $f_{s}^{\#}(x)$ belongs in the image of $F_{H, \varepsilon_{0}}: K_{i}\left(\Gamma_{H}\right)_{\varepsilon_{0}} \rightarrow K_{i}\left(\Gamma_{H}\right)$. Let $\delta$ be the control of the element $x$. Then, for all integers $s$ such that $s>\delta / \varepsilon_{0}$ and $s \equiv 1 \bmod \left(\left|G_{\Gamma}\right|^{2}\right), f_{s}^{\#}(x)$ is $\varepsilon_{0}$-controlled, i.e. $f_{s}^{\#}(x) \in \operatorname{Im} F_{H, \varepsilon_{0}}$. 


\section{A VANISHING THEOREM FOR THE LOWER $K$-THEORY}

In this section we will prove Theorem 5.2 below, for the controlled lower $K$-theory of crystallographic groups. The vanishing theorem, 5.3, follows as a direct consequence. We first explain the class of crystallographic groups for which 5.2 and 5.3 are proved. Let $\Gamma$ denote a fixed crystallographic group, $\Delta$ the group constructed in (2.1) and $j$ the epimorphism $\Gamma \rightarrow \Delta$.

Definition 5.1. We will say that $\Gamma$ satisfies "hypothesis $\mathscr{S}$ " if the following two conditions hold:

(i) Each finite subgroup $H$ of $\Gamma / A_{C}$ is the image of a finite subgroup of $\Gamma$ under the natural epimorphism $\Gamma \rightarrow \Gamma / A_{C}$. Here $A_{C}$ is the sum of all the 1-dimensional $G$-submodules of the translation subgroup of $\Gamma$.

(ii) If $\gamma \in \Gamma, a \in A$ and $\gamma a=a^{-1} \gamma$, then either $a=1$ or $\gamma$ has infinite order.

Next we briefly recall the definition of the groups $N^{j} K_{i}(\Gamma)$ which appear in the statement of Theorem 5.3. Actually, the functors $N^{j} K_{i}$ are derived from $K_{i}$ in the following formal way (see Bass [1]): Let $R:$ Rings $\rightarrow A b e l$ be a functor from the category of rings and ring homomorphisms to the category of abelian groups. Define $N F(R)$ for a ring $R$ as the kernel of the map $F(R([t])) \rightarrow$ $F(R)$, induced by the augmentation $R[t] \rightarrow R$. For $F=K_{i}$ and $R=\mathbb{Z} \Gamma$ we get the definition of the groups $N K_{i}(\Gamma)$ and we have the following identification: $K_{i}(\Gamma) \oplus N K_{i}(\Gamma) \approx K_{i}(\mathbb{Z} \Gamma[t])$. Moreover the fundamental theorem of algebraic $K$-theory holds in all dimensions; i.e.

$$
K_{i}\left(\mathbb{Z} \Gamma\left[t, t^{-1}\right]\right) \approx K_{i}(\Gamma) \oplus K_{i-1}(\Gamma) \oplus N K_{i}(\Gamma) \oplus N K_{i}(\Gamma) .
$$

The groups $N^{j} K_{i}(\Gamma)$ for $j \geq 1$ are defined inductively by applying the above definition for $F=N^{j-1} K_{i}$.

Theorem 5.2. Let $\Gamma$ be a crystallographic group which satisfies "hypothesis $\mathscr{S}$ ". Then the inclusion map induces an isomorphism $K_{i}(\Gamma)_{c} \stackrel{\approx}{\rightarrow} K_{i}(\Gamma \times \mathbb{Z})_{c}$ for all $i \leq-1$.

As a direct consequence of the above theorem together with 4.1, we have the following:

Theorem 5.3. If $\Gamma$ is a crystallographic group which satisfies "hypothesis $\mathscr{S}$ ", then $N^{j} K_{i}(\Gamma)=0$ if $i=-1$ and $j>0$, or if $i \leq-2$ and $j=0$.

Throughout this section $\Gamma$ will denote a crystallographic group as in Definition 5.1. For the proof of 5.2 we will use again Quinn's spectral sequence

$$
E_{\Gamma}: E_{j, i-j}^{2}=H_{j}^{G}\left(M_{\Delta} ; \pi_{i-j} \mathbb{K}\left(p^{-1}(x)\right)\right) \Rightarrow K_{i}(\Gamma)_{c},
$$

as described in the previous section.

By Lemma 3.4, we know explicitly the coefficient groups for the spectral sequence $E_{j, i-j}^{2}=H_{j}^{G}\left(M_{\Delta} ; \pi_{i-j} \mathbb{K}\left(p^{-1}(x)\right)\right) \Rightarrow K_{i}(\Gamma)_{c}$, in the case where $\Gamma$ is a crystallographic group which satisfies "hypotheses $\mathscr{S}$ ":

$$
\pi_{i} \mathbb{K}\left(p^{-1}(x)\right) \approx K_{i}\left(\pi_{1}\left(p^{-1}(x)\right)\right) \approx K_{i}\left(A_{C} \times G_{x}\right) .
$$

Proposition 5.4. If $G$ is a finite group and $A$ a free abelian group of finite rank, then the inclusion $G \rightarrow A \times G$ induces an isomorphism

$$
K_{i}(G) \stackrel{\approx}{\rightarrow} K_{i}(A \times G)
$$


for all $i \leq-1$. In particular $K_{i}(A \times G)=0$ for all $i \leq-2$.

Proof of Theorem 5.2 (using (5.4). The construction of $\Delta$ for the crystallographic group $\Gamma \times \mathbb{Z}$ will be identical with the one for $\Gamma$, except that $\operatorname{rk}\left(A_{\Gamma \times \mathbb{Z}}\right)_{C}$ $=\operatorname{rk}\left(A_{C}\right)+1$. That is to say: the inclusion map $\Gamma \rightarrow \Gamma \times \mathbb{Z}$ induces an isomorphism $\Delta_{\Gamma} \rightarrow \Delta_{\Gamma \times \mathbb{Z}}$. This shows that the control space for the group $K_{i}(\Gamma \times \mathbb{Z})_{c}$ can be chosed to be $M_{\Delta} / G_{\Delta}$. Let $p_{\Delta \times \mathbb{Z}}: M_{\Gamma \times \mathbb{Z}} \times_{G} E G \rightarrow M_{\Delta} / G$ be the control map associated to $\Gamma \times \mathbb{Z}$. Then, the fundamental group of the stalk $\pi_{1}\left(p_{\Delta \times \mathbb{Z}}^{-1}(x)\right)$ is isomorphic to $A_{C} \times \mathbb{Z} \times G_{x}$. Proposition 5.4 asserts that, for each $x$ in $M_{\Delta} / G_{\Delta}$, the inclusion

$$
\pi_{1}\left(p^{-1}([x])\right) \rightarrow \pi_{1}\left(p^{-1}([x])\right) \times \mathbb{Z} \approx \pi_{1}\left(p_{\Delta \times \mathbb{Z}}^{-1}(x)\right)
$$

induces an isomorphism between the coefficient groups of the spectral sequences $E_{\Gamma}$ and $E_{\Gamma \times \mathbb{Z}}$. So, it induces an isomorphism between the $E^{2}$ terms of $E_{\Gamma}$ and $E_{\Gamma \times \mathbb{Z}}$. Therefore the inclusion $\Gamma \rightarrow \Gamma \times \mathbb{Z}$ induces an isomorphism of their abutments, which are $K_{i}(\Gamma)_{c}$ and $K_{i}(\Gamma \times \mathbb{Z})_{c}$, respectively. This completes the proof of Theorem 5.2.

The rest of the paper is devoted to the proof of 5.4. The proof is a generalization of Carter's results in [4]. In the next paragraph we mention a few definitions and facts about the $K$-theory of well-behaved rings which will be used in the proof (for details see [22]).

(5.5) Let $R$ be a Noetherian commutative integral domain and $K$ its quotient field. An $R$-lattice in a $K$-space $V$ is a finitely generated $R$-submodule $M$ in $V$ such that $\left.K \cdot M=\left\{\sum_{\text {finite }} a_{i} m_{i}\right) / a_{i} \in K, m_{i} \in M\right\}=V$. An $R$-order in a $K$-algebra $A$ is a subring $\Lambda$ of $A$, with the same identity element as $A$, such that $\Lambda$ is an $R$-lattice in $A$. A maximal $R$-order is an $R$-order which is not properly contained in any other $R$-order in $A$. For any integrally slosed Noetherian domain $R$, each $R$-order in $A$ is contained in a maximal order in $A$ and there exists at least one maximal order. However, maximal orders are not unique. If the ring $R$ is, in addition, a Dedekind domain, then maximal $R$-orders in any separable $K$-algebra are regular. Recall that a ring $\Lambda$ is regular if $\Lambda$ is noetherian and every finitely generated $\Lambda$-module has a finite projective resolution over $\Lambda$; a ring $\Lambda$ is quasiregular if there exists a nilpotent ideal $N$ such that $\Lambda / N$ is regular. For our purposes, this is the most important property of maximal orders because of the following theorem of Bass [1, $\S 10.1]$.

Theorem C. If $\Lambda$ is quasiregular, then $K_{i}(\Lambda)=0$ for all $i<0$.

Recall also that if $N$ is nilpotent in $R$, then $K_{0}(R) \approx K_{0}(R / N)$ (see [24]). We will also use Serre's theorem:

Theorem D (Serre). Let $\Lambda=\Lambda_{0} \oplus \Lambda_{1} \oplus \Lambda_{2} \oplus \cdots$ be any graded ring. Assume $\tilde{\Lambda}$ is regular, where $\tilde{\Lambda}$ is $\Lambda$ without the grading. Then the inclusion $\Lambda_{0} \rightarrow \tilde{\Lambda}$ induces an isomorphism $K_{i}\left(\Lambda_{0}\right) \rightarrow K_{i}(\tilde{\Lambda})$.

Now let $G$ be a finite group, $n=|G|$ and $A=K G$. The following lemma is a standard result in the theory of orders (see, for example, [25]).

Lemma $\mathbf{E}$. Let $\Lambda$ be any order in $K G$ which contains $R G$. Then $n \Lambda$ is contained in $R G$. Moreover, $R G$ is a maximal $R$-order if and only if $n$ is invertible in $R$. 
Proof of Proposition 5.4. Let $\Lambda$ be a maximal order in $\mathbb{Q} G$ containing $\mathbb{Z} G$. Define $M:=\mathbb{Z} A \otimes_{\mathbb{Z}} \Lambda$. Then we obviously have that $\mathbb{Z}(A \times G) \subseteq M \subseteq \mathbb{Q}(A \times G)$. Moreover $n M \subseteq \mathbb{Z}(A \times G)$ because, by Lemma $\mathrm{E}, n \Lambda \subseteq \mathbb{Z} G$ and the tensor product is taken over $\mathbb{Z}$. So we have the following cartesian square:

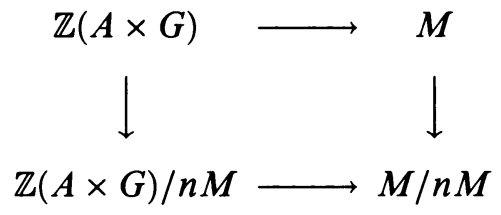

where $\mathbb{Z}\left(A \times G / n M \approx \mathbb{Z} A \otimes_{\mathbb{Z}} \mathbb{Z} G / n \Lambda\right.$ and $M / n M \approx \mathbb{Z} A \otimes_{\mathbb{Z}} \Lambda / n \Lambda$. The above diagram gives rise to a Mayer-Vietoris exact sequence in $K$-theory (see Milnor [15]) for $i \leq 2$, and we have the following commutative diagram:

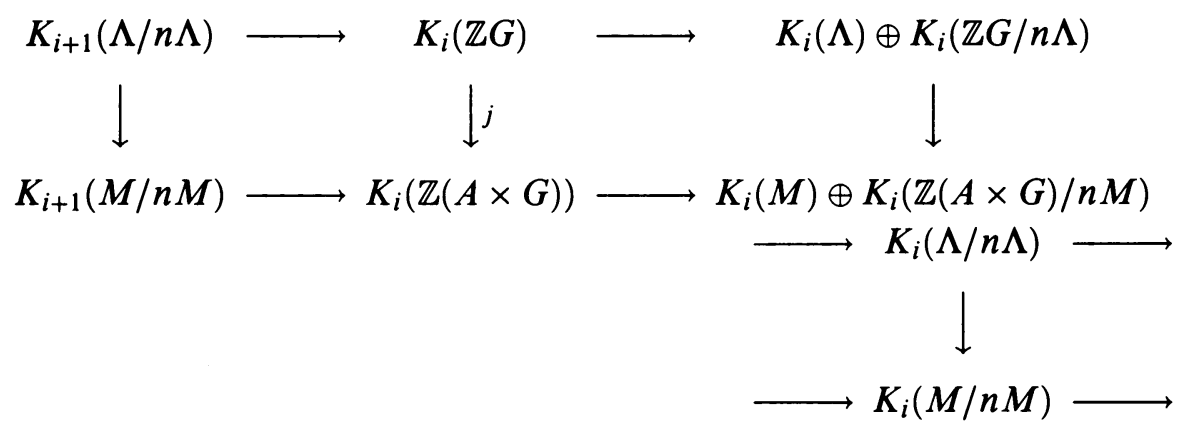

The top exact sequence in this diagram corresponds to the case $\operatorname{rk}(A)=0$.We can view $M$ as a graded ring with $\Lambda$ as the zero grading by means of the isomorphism $M \equiv \mathbb{Z} A \otimes_{\mathbb{Z}} \Lambda \approx \Lambda\left[t_{1}, t_{1}^{-1}, \ldots, t_{k}, t_{k}^{-1}\right]$, where $k$ is the rank of $A$. Then by Serre's theorem, since $\Lambda$ is regular, $K_{i}(M) \approx K_{i}(\Lambda)$ for all $i \leq 0$. The rings $\mathbb{Z} G / n \Lambda$ and $\Lambda / n \Lambda$ are finite and therefore quasiregular. Again by Serre's theorem we have $K_{i}(\mathbb{Z}(A \times G) / n M) \approx K_{i}(\mathbb{Z} G / n \Lambda)$ and $K_{i}(M / n M) \approx$ $K_{i}(\Lambda / n \Lambda)$, so that the maps

$$
\begin{gathered}
K_{i}(\Lambda) \oplus K_{i}(\mathbb{Z} G / n \Lambda) \rightarrow K_{i}(M) \oplus K_{i}(\mathbb{Z}(A \times G) / n M), \\
K_{i}(\Lambda / n \Lambda) \rightarrow K_{i}(M / n M)
\end{gathered}
$$

are isomorphisms for all $i \leq 0$. It then follows, by the 5-lemma, the map $j: K_{i}(\mathbb{Z} G) \rightarrow K_{i}(\mathbb{Z}(A \times G))$ is an isomorphism, for all $i \leq-1$.

\section{ACKNOWLEDGMENT}

These results are part of the doctoral dissertation of the author written under the direction of Professor Francis X. Connolly, to whom the author is grateful, at the University of Notre Dame.

\section{REFERENCES}

1. H. Bass, Algebraic K-theory, Benjamin, 1968.

2. G. Bredon, Equivariant cohomology theories, Lecture Notes in Math., vol. 34, SpringerVerlag, 1967.

3. D. Carter, Localization in lower algebraic K-theory, Comm. Algebra 8 (1980), 603-622.

4. , Lower K-theory of finite groups, Comm. Algebra 8 (1980), 1927-1937. 
5. T. A. Chapman, Controlled simple homotopy theory and applications, Lecture Notes in Math., vol. 1099, Springer, 1983.

6. M. Cohen, A course in simple homotopy theory, Springer-Verlag, New York, 1973.

7. __ Simplicial structure and transverse cellularity, Ann. of Math. (2) 85 (1967), 218-245.

8. F. Connolly and T. Kózniewski, Rigidity and crystallographic groups. I, Invent Math. 99 (1990), 25-48.

9. A. Dress, Induction and structure theorems for orthogonal representations of finite groups, Ann. of Math. (2) 102 (1975), 291-335.

10. D. Epstein and M. Shub, Expanding endomorphisms of flat manifolds, Topology 7 (1968), 139-141.

11. F. T. Farrell and W. C. Hsiang, The topological Euclidean space form problem, Invent. Math. 45 (1978), 181-192.

12. (1981), 308-324.

13. $\ldots$ Topological characterization of flat and almost flat Riemannian manifolds, Amer. J. Math. 105 (1983), 641-672.

14. D. Farkas, Crystallographic groups and their mathematics, Rocky Mountain J. Math. 11 (1971), 511-551.

15. J. Milnor, Introduction to algebraic K-theory, Ann. of Math. Stud., no. 72, Princeton Univ. Press, 1971.

16. __ Construction of universal bundles. II, Ann. of Math. (2) 63 (1956), 430-436.

17. —_ Whitehead torsion, Bull. Amer. Math. Soc. 72 (1966), 358-426.

18. F. Quinn, Ends of maps. II, Invent. Math. 68 (1982), 353-424.

19. (1985).

20. __ Geometric algebra and ends of maps, Lecture Notes of CBMS Conference Lectures at the University of Notre Dame.

21. A. Ranicki, Lower K- and L-theory, London Math. Soc. Lecture Note Ser., vol. 178, Cambridge Univ. Press, 1992.

22. I. Reiner, Maximal orders, Academic Press, 1975.

23. R. Stöcker, Whiteheadgruppe topologischer Räume, Invent. Math. 9 (1980), 271-278.

24. R. G. Swan, Algebraic K-theory, Lecture Notes in Math., vol. 76, Springer-Verlag, 1968.

25. R. G. Swan and E. G. Evans, K-theory of finite groups and orders, Lecture Notes in Math., vol. 149, Springer-Verlag, 1970.

26. G. Tsapogas, K-theory of crystallographic groups, $\mathrm{Ph} . \mathrm{D}$. Dissertation, University of Notre Dame, 1989.

27. M. Yamasaki, Surgery groups of crystallographic groups, Invent. Math. 88 (1987), 571-602.

28. J. Wolf, Spaces of constant curvature, McGraw-Hill, 1967.

29. F. T. Farrell and L. E. Jones, Isomorphism conjectures in algebraic $\mathrm{K}$-theory, J. Amer. Math. Soc. 6 (1993), 249-297.

Department of Mathematics, University of the Aegean, Karlovassi 83200 Samos, GREECE

E-mail address: gtsap@pythagoras. aegean .ariadne-t.gr 